\title{
A NEW PROOF AND A GENERALIZATION OF AN INEQUALITY OF BOHR
}

\section{LARS HÖRMANDER}

Introduction. Let $f(x)$ be a real function of the real variable $x$ with a bounded derivative. If $f(x)$ is integrable its Fourier transform is defined by

$$
\hat{f}(\xi)=\int_{-\infty}^{+\infty} f(x) e^{-2 \pi i x \xi} d x,
$$

and the smallest closed set outside which $\hat{f}(\xi)$ vanishes is called the spectrum of $f(x)$. When $f(x)$ is not integrable the spectrum is defined according to Schwartz [7]. The definition is also stated explicitly on page 39.

Suppose that $f(x)$ has no spectrum in $(-\Lambda, \Lambda)$. Then we have

$$
\sup _{-\infty<x<\infty}|f(x)| \leqq(4 \Lambda)^{-1} \sup _{-\infty<x<\infty}\left|f^{\prime}(x)\right|,
$$

and in fact the constant $(4 \Lambda)^{-1}$ is the best possible. The inequality (1) was given by Bohr [2] for almost periodic $f(x)$ with a proof based on the theory of analytic functions.

Iteration of (1) gives the inequality

$$
\sup _{-\infty<x<\infty}|f(x)| \leqq \Lambda^{-n} t_{n} \sup _{-\infty<x<\infty}\left|f^{(n)}(x)\right|
$$

with $t_{n}=4^{-n}$. With methods from the theory of real functions, Favard [3] found that the best possible value of $t_{n}$ is

$$
t_{n}=8(2 \pi)^{-(n+1)}\left[1+(-3)^{-(n+1)}+5^{-(n+1)}+(-7)^{-(n+1)}+\ldots\right] .
$$

We shall here give the following generalization: If $f(x)$ is real and

then

$$
-M_{1} \leqq f^{(n)}(x) \leqq M_{2},
$$

$$
-\Lambda^{-n} \mu_{1}^{(n)}\left(M_{1}, M_{2}\right) \leqq f(x) \leqq \Lambda^{-n} \mu_{2}^{(n)}\left(M_{1}, M_{2}\right),
$$

where $\mu_{1}^{(n)}$ and $\mu_{2}^{(n)}$ denote the best possible constants and will be determined explicitly. In particular,

Received January 12, 1954. 


$$
\mu_{1}^{(1)}=\mu_{2}^{(1)}=\frac{M_{1} M_{2}}{2\left(M_{1}+M_{2}\right)},
$$

that is, one fourth of the harmonic mean of $M_{1}$ and $M_{2}$. It is interesting that the $\mu_{i}^{(n)}$ have finite limiting values when one of the bounds $M_{1}$ and $M_{2}$ tends to infinity. This leads to an estimate even in the case when (3) is one-sided, which seems to be an essentially new theorem.

We first restrict ourselves to periodic functions $f(x)$. The extension to the general case is achieved by an improvement of an approximation method due to Lewitan [5], who was the first to prove (1) for a general $f(x)$.

The periodic case. ${ }^{1}$ Let $f(x)$ be a real function with period 1 which is $n-1$ times continuously differentiable and satisfies the conditions:

(A) The Fourier coefficients $c_{k}=\int_{0}^{1} f(x) e^{-2 \pi i k x} d x$ vanish for $|k|<m$.

$$
-M_{1} \leqq \frac{f^{(n-1)}(x)-f^{(n-1)}(y)}{x-y} \leqq M_{2},
$$

where $M_{1}$ and $M_{2}$ are finite positive numbers.

The condition (B) is, of course, equivalent to:

$\left(\mathrm{B}^{\prime}\right)\left\{\begin{array}{l}f^{(n-1)}(x) \text { is absolutely continuous and }-M_{1} \leqq f^{(n)}(x) \leqq M_{2} \text { almost } \\ \text { everywhere. }\end{array}\right.$

We start by constructing a function which satisfies (A) and (B) and which will prove to have extremal properties. Let $h_{0}(x)=h_{0}\left(x ; M_{1}, M_{2}\right)$ be the function with period 1 defined by

$$
\begin{aligned}
& h_{0}(x)=-M_{1} \quad \text { for } \quad \frac{-M_{2}}{2\left(M_{1}+M_{2}\right)}<x<\frac{M_{2}}{2\left(M_{1}+M_{2}\right)}, \\
& h_{0}(x)=M_{2} \quad \text { for }\left\{\begin{array}{l}
\frac{-M_{1}}{2\left(M_{1}+M_{2}\right)}<x-\frac{1}{2}<\frac{M_{1}}{2\left(M_{1}+M_{2}\right)}, \text { that is, } \\
\frac{M_{2}}{2\left(M_{1}+M_{2}\right)}<x<\frac{-M_{2}}{2\left(M_{1}+M_{2}\right)}+1 .
\end{array}\right.
\end{aligned}
$$

Obviously $\int_{0}^{1} h_{0}(x) d x=0$. Hence the indefinite integral of $h_{0}(x)$ is peri-

1 This section was written in 1951 and presented at a meeting of the Danish Mathematical Society at Professor Jessen's suggestion. I take this opportunity to thank Professor Jessen for his encouraging interest in my paper. My thanks are also due to C. Hyltén-Cavallius and T. Herlestam for valuable criticism on various details. 
odic, and we can choose the integration constant such that the mean value is 0 . Call this integral $h_{1}(x)$. By integrating successively in this manner we find $h_{n}(x)=h_{n}\left(x ; M_{1}, M_{2}\right)$, the $n^{\text {th }}$ periodic integral of $h_{0}(x)$ with vanishing mean value.

It is easy to express $h_{n}(x)$ in terms of the periodic functions $\bar{B}_{n}(x)$, which have the period 1 and in the interval $(0,1)$ coincide with the Bernoulli polynomials $B_{n}(x)$ (cf. Nörlund [6, Kapitel 2-3]). Since $\bar{B}_{1}(x)=x-\frac{1}{2}$ for $0<x<1$ we find that

$$
\begin{aligned}
& h_{0}\left(x ; M_{1}, M_{2}\right) \\
& \quad=\left(M_{1}+M_{2}\right)\left\{\bar{B}_{1}\left(x+\frac{M_{2}}{2\left(M_{1}+M_{2}\right)}\right)-\bar{B}_{1}\left(x-\frac{M_{2}}{2\left(M_{1}+M_{2}\right)}\right)\right\} .
\end{aligned}
$$

From the well-known facts that the mean value of $\bar{B}_{n}(x)$ is 0 and $\bar{B}_{n}{ }^{\prime}=n \bar{B}_{n-1}$ it now follows that

$$
\begin{aligned}
& h_{n}\left(x ; M_{1}, M_{2}\right) \\
& \quad=\frac{M_{1}+M_{2}}{(n+1) !}\left\{\bar{B}_{n+1}\left(x+\frac{M_{2}}{2\left(M_{1}+M_{2}\right)}\right)-\bar{B}_{n+1}\left(x-\frac{M_{2}}{2\left(M_{1}+M_{2}\right)}\right)\right\} .
\end{aligned}
$$

The function $m^{-n} h_{n}\left(m x ; M_{1}, M_{2}\right)$ satisfies (A) and (B). That (A) is fulfilled is obvious because the period is $m^{-1}$ and the mean value vanishes. (B') follows from the fact that the $n^{\text {th }}$ derivative is $h_{0}(m x)$.

Theorem 1. If $f(x)$ satisfies $(\mathrm{A})$ and $(\mathrm{B})$, then

$$
\min _{x}\left(m^{-n} h_{n}\left(m x ; M_{1}, M_{2}\right)\right) \leqq f(x) \leqq \max _{x}\left(m^{-n} h_{u}\left(m x ; M_{1}, M_{2}\right)\right),
$$

or, equivalently,

where

$$
-m^{-n} \mu_{1}^{(n)}\left(M_{1}, M_{2}\right) \leqq f(x) \leqq m^{-n} \mu_{2}^{(n)}\left(M_{1}, M_{2}\right)
$$

$$
-\mu_{1}^{(n)}=\min _{x} h_{n}(x), \quad \mu_{2}^{(n)}=\max _{x} h_{n}(x) .
$$

There is inequality for all $x$ unless $f(x)=m^{-n} h_{n}(m x+\delta)$, where $\delta$ is a constant.

The proof depends on a study of the zeros of $f(x)$. By a zero we shall mean a point or a closed interval in which $f(x)$ vanishes without being identically zero in any enclosing interval. By the number of zeros of a periodic function we mean the number of zeros in a period. Analogously a maximum or minimum can be a point or an interval. We first prove 
Lemma 1. If the Fourier coefficients $c_{k},|k|<m$, of a real continuous function $g(x) \neq 0$ with period 1 all vanish, then $g(x)$ has at least $2 m$ zeros.

Proof. Because $\int_{0}^{1} g(x) d x=0, g(x)$ has at least two zeros. Suppose that it has less than $2 m$ zeros. Between two consecutive zeros $g(x)$ has a constant sign. Now take points $0 \leqq x_{1}<x_{2}<\ldots<x_{2 l}<1$, one in every zero at which the sign of $g(x)$ changes. Let $P(x)$ be a trigonometric polynomial of order $l<m$ which has simple zeros at $x_{i}$ and hence changes its sign at these points. Then $P(x) g(x)$ has a constant sign but $\int_{0}^{1} P(x) g(x) d x=0$ by the assumption. Thus $P(x) g(x)$ and hence $g(x)$ must vanish identically, which gives a contradiction.

For future use we note that it also follows from the proof that if $g(x)$ has precisely $2 m$ zeros then the sign of $g(x)$ must change at every one of them.

We can now prove theorem 1 for $n=1$. Let $f(x)$ satisfy (A) and (B) for $n=1$. Put

$$
g(x)=m^{-1} h_{1}(m x)-f(x)
$$

and suppose that $g(x) \neq 0$. From (B) it follows that $g(x)$ and $m^{-1} h_{1}(m x)$ increase and decrease simultaneously because the slope of the latter function is always $-M_{1}$ or $M_{2}$. Hence $g(x)$ has maxima and minima for

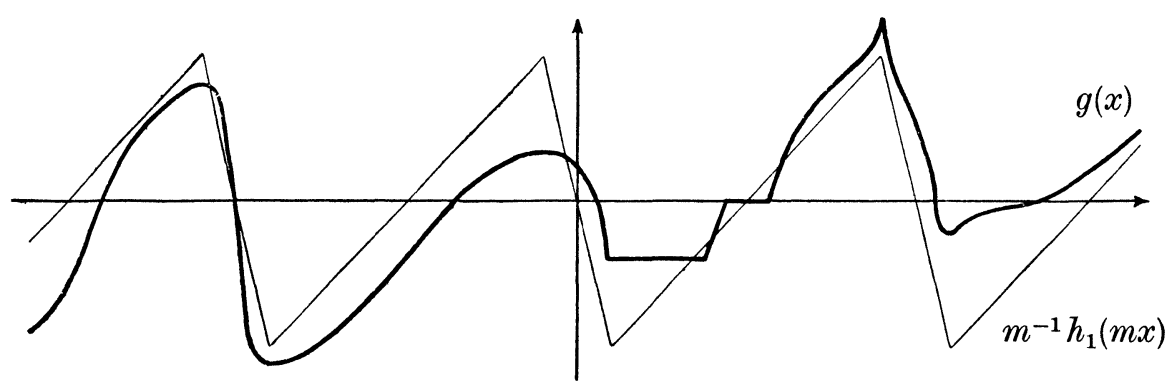

Fig. 1.

the same values of $x$ as $m^{-1} h_{1}(m x)$. Furthermore, $g(x)$ has at most one zero in every interval where $m^{-1} h_{1}(m x)$ is monotone, hence in all at most $2 m$. But from (A) it follows that the assumptions of lemma 1 are satisfied by $g(x)$ so that $g(x)$ must have at least $2 m$ zeros. Hence by the remark at the end of the proof of lemma $1, g(x)$ changes its sign once in every interval of linearity of $m^{-1} h_{1}(m x)$. Thus it follows that the maxima of $g(x)$ are positive and the minima negative, that is, $f(\xi)<$ $m^{-1} h_{1}(m \xi)$ if $\xi$ is a maximum point of $m^{-1} h_{1}(m x)$ and $f(\eta)>m^{-1} h_{1}(m \eta)$ 
if $\eta$ is a minimum point of that function. Since the conditions (A) and $(\mathrm{B})$ are also satisfied by the translated functions $f(x+\delta)(\delta=$ constant) the theorem follows if we apply our result to these functions.

To prove theorem 1 for $n>1$ we need the following

Lemma 2. Let $g(x)$ be a real function with period 1 and let the Fourier coefficients $c_{k}=0,|k|<m$. Suppose that $g(x)$ is $v$ times continuously differentiable $(v \geqq 1)$ and that $g^{(v)}(x)$ has exactly $2 m$ zeros. Then $g(x)$ has precisely $2 m$ zeros and $2 m$ extrema, $m$ positive maxima and $m$ negative minima. The extrema are the only zeros of $g^{\prime}(x)$.

Proof. By Rolle's theorem, $g^{\prime}(x)$ has at least one zero between two zeros of $g(x)$ so that $g^{\prime}(x)$ must in all have at least as many zeros as $g(x)$. Hence the number of zeros of $g^{(v)}(x)$, that is $2 m$, is at least as great as the number of zeros of $g(x)$. But, according to lemma $1, g(x)$ has at least $2 m$ zeros and hence precisely $2 m$ zeros. It was remarked at the end of the proof of lemma 1 that the sign of $g(x)$ must then change in all the zeros. Hence $g(x)$ has at least $m$ positive maxima and $m$ negative minima, but since $g^{\prime}(x)$ cannot have more than $2 m$ zeros (Rolle's theorem again) these are the only extrema.

Note that $h_{n}(m x)$ satisfies the assumptions of this lemma (with $\nu=n-1$, for example).

We now prove the theorem for $n>1$. Suppose it has already been proved for $n-1$. Let $f(x)$ satisfy (A) and (B) and attain its maximum at a point $\xi$. We may suppose that $\xi$ is also a maximum point of $m^{-n} h_{n}(m x)$ (otherwise we consider $f(x+\delta)$ for a suitable $\delta$ ). Introduce

$$
g(x)=m^{-n} h_{n}(m x)-f(x)
$$

and suppose that $g(x) \neq 0$. In the proof for $n=1$ we have shown that $g^{(n-1)}(x)=m^{-1} h_{1}(m x)-f^{(n-1)}(x)$ has exactly $2 m$ zeros. Hence, by lemma $2, g(x)$ has $2 m$ extrema, $m$ of which are positive maxima and $m$ negative minima. Among these is $\xi$, because $g^{\prime}(\xi)=0$. Thus in order to prove that $g(\xi)>0$ we have just to show that $\xi$ is a maximum point.

From the induction assumption it follows that $g^{\prime}(x)$ and $\left(m^{-n} h_{n}(m x)\right)^{\prime}=$ $=m^{-(n-1)} h_{n-1}(m x)$ have the same signs at the extrema of the latter function. Hence $g^{\prime}(x)$ has at least one zero between two such extrema and as $g^{\prime}(x)$ has only $2 m$ zeros there can never be more than one. Now $\xi$ is a maximum point of $m^{-n} h_{n}(m x)$ so that the extreme values of $m^{-(n-1)} h_{n-1}(m x)$ next to the left and right of $\xi$ are positive and negative, respectively. Hence $g^{\prime}(x)$ is positive to the left and negative to the right of $\xi$, that is, $\xi$ is a maximum. This completes the proof of theorem 1 as 
far as the maximum of $f(x)$ is concerned. The minimum is treated analogously.

We now study the case of a one-sided estimate, that is, we consider $n-2$ times continuously differentiable functions $f(x)$ such that $f^{(n-2)}(x)$ is absolutely continuous and

$$
\frac{f^{(n-1)}(x)-f^{(n-1)}(y)}{x-y} \leqq M_{2}, \quad\left(0<M_{2}<\infty\right) .
$$

This means that $f^{(n-1)}(x)-M_{2} x$ is decreasing, so that $f^{(n-1)}(x)$ is of bounded variation. Put

$$
h_{n}\left(x ; \infty, M_{2}\right)=M_{2} \frac{\bar{B}_{n}(x)}{n !}=\lim _{M_{1} \rightarrow \infty} h_{n}\left(x ; M_{1}, M_{2}\right)
$$

and define $\mu_{i}^{(n)}\left(\infty, M_{2}\right)$ according to (7) by means of this function. We shall show that theorem 1 is still valid, in other words: if $f(x)$ satisfies (A) and $\left(B_{\infty}\right)$ then $(6)$ is valid.

This can be proved by using the same argument as before provided that we also call $x$ a zero of $f(x)$ if $f(x-0)$ and $f(x+0)$ have opposite signs or if one of them vanishes. If we are not interested in the cases of equality it also follows by regularization from the results already obtained. For take $\varphi(x) \geqq 0$ periodic and infinitely differentiable with $\int_{0}^{1} \varphi(x) d x=1$. Consider

$$
f_{\varphi}(x)=\varphi * f(x)=\int_{0}^{1} \varphi(x-t) f(t) d t=\int_{0}^{1} f(x-t) \varphi(t) d t .
$$

The convolution $f_{\varphi}(x)$ satisfies $\left(\mathrm{B}_{\infty}\right)$. Since all of its derivatives are continuous, we have $f_{\varphi}^{(n)}(x) \geqq-M_{1}$ for some $M_{1}$. Using

$$
\int_{0}^{1} f_{\varphi}(x) e^{-2 \pi i k x} d x=\left\{\int_{0}^{1} f(x) e^{-2 \pi i k x} d x\right\}\left\{\int_{0}^{1} \varphi(x) e^{-2 \pi i k x} d x\right\}=0
$$

if $|k|<m$, we obtain

$-\frac{\mu_{1}{ }^{(n)}\left(\infty, M_{2}\right)}{m^{n}}<-\frac{\mu_{1}^{(n)}\left(M_{1}, M_{2}\right)}{m^{n}} \leqq f_{p}(x) \leqq \frac{\mu_{2}{ }^{(n)}\left(M_{1}, M_{2}\right)}{m^{n}}<\frac{\mu_{2}^{(n)}\left(\infty, M_{2}\right)}{m^{n}}$.

Here the first and last inequalities follow from the facts that $\mu_{i}^{(n)}\left(M_{1}, M_{2}\right)$, being the best possible constants in (6), must increase with $M_{1}$ and that the limits when $M_{1} \rightarrow \infty$ are $\mu_{i}^{(n)}\left(\infty, M_{2}\right)$. If we now let $\varphi$ converge towards the Dirac measure $\delta(0)$, then $f_{\varphi}(x)$ converges to $f(x)$ at every point of continuity and we have obtained the desired estimate. 
The general case. Consider a real function $f(x)$ which is $n-1$ times continuously differentiable and satisfies the conditions:

(A) The spectrum of $f(x)$ is situated outside the open interval $(-\Lambda, \Lambda)$.

(B) \{

$$
-M_{1} \leqq \frac{f^{(n-1)}(x)-f^{(n-1)}(y)}{x-y} \leqq M_{2},
$$

where $M_{1}$ and $M_{2}$ are finite positive numbers.

Note that it follows from (B) that $f(x)$ is continuous and does not increase more rapidly than $x^{n}$ at infinity so that the Fourier transform $\hat{f}$ of $f$ exists in the sense of Schwartz [7]. The spectrum of $f$ is by definition the support of $\hat{f}$. Thus assumption (A) means explicitly that $\int_{-\infty}^{+\infty} f(x) \psi(x) d x=0$ if $\psi(x) \in \mathcal{S}$ and $\hat{\psi}(\xi)$ vanishes outside a compact set in $(-\Lambda, \Lambda)$. ( $\mathcal{S}$ is the class of all infinitely differentiable functions which vanish at infinity together with all their derivatives more rapidly than any inverse power of $x$.)

If $f(x)$ has a Fourier transform or is an inverse Fourier transform in a classical sense this definition of spectrum is equivalent to the classical one. Thus, for example, if $f(x)$ is a function with period 1 we have (with convergence in the mean) $f(x)=\sum_{-\infty}^{+\infty} c_{k} e^{2 \pi i k x}, \quad c_{k}=\int_{0}^{1} f(x) e^{-2 \pi i k x} d x$, so that $\hat{f}$ is the measure having the mass $c_{k}$ at $\xi=k, k=0, \pm 1, \pm 2, \ldots$, and no masses elsewhere. Hence, in this case assumption (A) means that $c_{k}=0$ if $|k|<\Lambda$ which coincides with the assumption (A) of p. 34 . Theorem 1 is therefore a special case of the following

Theorem 2. If $f(x)$ satisfies (A) and (B), then

$$
-\Lambda^{-n} \mu_{1}^{(n)}\left(M_{1}, M_{2}\right) \leqq f(x) \leqq \Lambda^{-n} \mu_{2}^{(n)}\left(M_{1}, M_{2}\right),
$$

where $\mu_{i}^{(n)}$ are defined in (7).

Since this theorem is invariant if we substitute $f(c x)$ for $f(x) \quad(c=$ constant $>0$ ), it is true for any periodic function $f(x)$ according to theorem 1 . To prove it generally we shall use a method of approximating bounded functions by periodic functions.

We first consider continuous functions $\varphi(x)$ with the properties

$$
\varphi(x) \geqq 0, \quad \sum_{-\infty}^{+\infty} \varphi(x+n) \leqq 1, \quad \varphi(0)=1 .
$$

An example of such a function is $\varphi(x)=(\pi x)^{-2} \sin ^{2} \pi x$. In this case we have even $\Sigma_{-\infty}^{+\infty} \varphi(x+n) \equiv 1$ which follows from a well-known expansion of $1 / \sin ^{2} \pi x$. This identity also follows if we observe that 
$\hat{\varphi}(\xi)=1-|\xi|$ for $|\xi| \leqq 1$ and vanishes elsewhere, and we apply Poisson's formula. Lewitan's procedure in [5] may be shown to be substantially equivalent to ours with this special choice of $\varphi(x)$.

A more general example of such a function is $\varphi(x)=\alpha(x)(\pi x)^{-2} \sin ^{2} \pi x$ where $\alpha(x)$ is a continuous function with $0 \leqq \alpha(x) \leqq \alpha(0)=1$. This inequality is obviously satisfied if $\alpha(x)=(\psi(x))^{2}$ where $\psi(x)$ is the inverse Fourier transform of a positive, even, integrable function $\hat{\psi}(\xi)$ such that

$$
\int_{-\infty}^{+\infty} \hat{\psi}(\xi) d \xi=1 .
$$

If $\hat{\psi}(\xi)$ is infinitely differentiable and vanishes outside a bounded set, we have $\hat{\psi}(\xi) \in \mathscr{S}$ and consequently $\psi(x)$ is also in $\mathcal{P}$ (cf. Schwartz [7], p. 105). Hence in this case $\varphi(x) \in \mathscr{S}$ and $\hat{\varphi}(\xi)$ vanishes outside a bounded set because it is the convolution of $\hat{\psi}(\xi) * \hat{\psi}(\xi)$ and the function which is $1-|\xi|$ for $|\xi| \leqq 1$ and vanishes elsewhere.

Now take a fixed function $\varphi(x)$ having the properties (9). If $g(x)$ is a bounded function we set

$$
g_{h}(x)=\sum_{-\infty}^{+\infty} \varphi(h x+n) g\left(x+n h^{-1}\right) .
$$

It is evident that the series converges and that $g_{h}(x)$ has the period $h^{-1}$.

Lemma 3. If $-M_{1} \leqq g(x) \leqq M_{2}$, then $-M_{1} \leqq g_{h}(x) \leqq M_{2}$, and $g_{h}(x)$ tends to $g(x)$ as $h \rightarrow 0$, uniformly on every bounded set.

Proof. The first part of the lemma follows at once from the first two properties in (9). We may write

$$
\begin{aligned}
g_{h}(x)-g(x) & =(\varphi(h x)-1) g(x)+\sum_{n \neq 0} \varphi(h x+n) g\left(x+n h^{-1}\right), \\
\left|g_{h}(x)-g(x)\right| & \leqq M(1-\varphi(h x))+M \sum_{n \neq 0} \varphi(h x+n) \\
& \leqq M(1-\varphi(h x))+M(1-\varphi(h x))=2 M(\varphi(0)-\varphi(h x)),
\end{aligned}
$$

and because $\varphi(x)$ is continuous for $x=0$ the uniform convergence on every bounded set follows.

We now calculate the Fourier coefficients of $g_{h}(x)$ :

$$
\begin{aligned}
h \int_{0}^{h^{-1}} g_{h}(x) e^{-2 \pi i k h x} d x & =\sum_{-\infty}^{+\infty} h \int_{0}^{h^{-1}} \varphi\left(h\left(x+n h^{-1}\right)\right) g\left(x+n h^{-1}\right) e^{-2 \pi i k h x} d x \\
& =h \int_{-\infty}^{+\infty} \varphi(h x) g(x) e^{-2 \pi i k h x} d x .
\end{aligned}
$$


To our assumption (9) concerning $\varphi(x)$ we now add that $\varphi(x) \in \mathcal{f}$ and that $\hat{\varphi}(\xi)$ shall vanish outside a bounded set or, equivalently, that $\varphi$ shall be an analytic function of exponential type. That there exist functions satisfying these conditions and the conditions (9) has been shown by an example above. Denote by $A$ a number such that $\hat{\varphi}(\xi)=0$ for $|\xi| \geqq A$.

The Fourier transform of $h \varphi(h x) e^{-2 \pi i k h x}$ is $\hat{\varphi}((\xi+k h) / h)=\hat{\varphi}\left(\xi h^{-1}+k\right)$. It vanishes outside an interval contained in $(-\Lambda, \Lambda)$ if $|k h|<\Lambda-A h$. Hence

Lemma 4. $g_{h}(x)$ has no spectrum in $(-(\Lambda-A h),(\Lambda-A h))$ if $g(x)$ has no spectrum in $(-\Lambda, \Lambda)$.

We can now prove theorem 2. The function $f^{(n)}(x)$ satisfies the conditions imposed on $g(x)$ above. Denote its periodic approximating function (10) by $f_{h}^{(n)}(x)$. According to lemma 3 we have $-M_{1} \leqq f_{h}^{(n)}(x) \leqq M_{2}$. From lemma 4 it follows that $f_{h}^{(n)}(x)$ has no spectrum in $|\xi| \leqq \Lambda-A h$. Hence, if $h$ is so small that $\Lambda-A h>0$, there exists a periodic function $f_{h}(x)$ with vanishing mean value, the $n^{\text {th }}$ derivative of which is $f_{h}^{(n)}(x)$. Theorem 1 gives

$$
-\frac{\mu_{1}^{(n-k)}\left(M_{1}, M_{2}\right)}{(\Lambda-A h)^{n-k}} \leqq f_{h}^{(k)}(x) \leqq \frac{\mu_{2}^{(n-k)}\left(M_{1}, M_{2}\right)}{(\Lambda-A h)^{n-k}}, \quad k=0, \ldots, n-1 .
$$

Now it just remains to prove that $f_{h}(x) \rightarrow f(x)$ as $h \rightarrow 0$ and the theorem will be proved. Choose a sequence $h_{i} \rightarrow 0$ such that $f_{h_{i}}{ }^{(k)}(0)$ converges for $0 \leqq k<n$ as $i \rightarrow \infty$. This is possible because we have obtained a bound for $f_{h}^{(k)}$ above. But then it follows from

$$
f_{h}(x)=f_{h}(0)+\ldots+\frac{x^{n-1}}{(n-1) !} f_{h}^{(n-1)}(0)+\int_{0}^{x} \frac{(x-t)^{n-1}}{(n-1) !} f_{h}^{(n)}(t) d t,
$$

that $f_{h_{i}}(x)$ converges uniformly on every compact set to a function $F(x)$ whose $n^{\text {th }}$ derivative is $f^{(n)}(x)$. Hence $F(x)-f(x)$ is a polynomial of degree at most $n-1$. Since the functions $f_{h_{i}}$ are uniformly bounded and have no spectrum in $|\xi|<\Lambda-A h \rightarrow \Lambda, F$ has no spectrum in $(-\Lambda, \Lambda)$. Hence $F-f$ cannot have any spectrum in $(-\Lambda, \Lambda)$ either, which implies that $F-f=0$ because the Fourier transform of a polynomial is supported by the origin. Since we can select from every sequence $f_{h_{i}}, h_{i} \rightarrow 0$, a subsequence which converges to $f$, it follows that $f_{h}(x) \rightarrow f(x)$ uniformly on every compact set as $h \rightarrow 0$.

We shall now study one-sided estimates, that is, we consider $n-2$ times continuously differentiable functions $f(x)$ such that $f^{(n-2)}(x)$ is absolutely continuous, and 
$\left(\mathrm{A}_{\infty}\right)\left\{\begin{array}{l}f(x) \text { is slowly increasing, that is }|f(x)|<C\left(1+x^{2}\right)^{N} \text { for some } C \text { and } \\ N \text {, and its spectrum is situated outside }(-\Lambda, \Lambda)\end{array}\right.$ $\left(\mathrm{B}_{\infty}\right)$

$$
\frac{f^{(n-1)}(x)-f^{(n-1)}(y)}{x-y} \leqq M_{2}, \quad\left(0<M_{2}<\infty\right) .
$$

As we have pointed out on page $38,\left(\mathrm{~B}_{\infty}\right)$ implies that $f^{(n-1)}(x)$ is of locally bounded variation and hence in particular locally integrable. Observe, however, that now it does not follow from $\left(\mathrm{B}_{\infty}\right)$ that $f(x)$ is slowly increasing so we have to include this in the assumption $\left(A_{\infty}\right)$ in order that we should be able to define the spectrum.

We shall prove that theorem 2 is still valid in this case. The proof is a reduction in two steps to the case of two-sided estimates.

Suppose first that $f, f^{\prime}, \ldots, f^{(n)}$ all exist and are continuous and that $\left|f^{(n)}(x)\right| \leqq C\left(1+x^{2}\right)^{N}$ for some $C$ and $N$. Let $\alpha(x)$ be a function in $\mathcal{S}$ such that $0 \leqq \alpha(x) \leqq \alpha(0)=1$ and $\hat{\alpha}(\xi)$ vanishes outside $(-1,1)$, for example. Such functions exist; in fact an example was constructed on page 40.

We shall show that $\alpha(h x) f^{(n)}(x)$ has no spectrum in $(-(\Lambda-h),(\Lambda-h))$. Take $\psi(x) \in \mathcal{S}$ such that $\hat{\psi}(\xi)=0$ outside a compact set in the interval $(-(\Lambda-h),(\Lambda-h))$. To prove that

$$
\int_{-\infty}^{+\infty}\left(\alpha(h x) f^{(n)}(x)\right) \psi(x) d x=\int_{-\infty}^{+\infty} f^{(n)}(x)(\alpha(h x) \psi(x)) d x=0
$$

we have just to show that the Fourier transform of $\alpha(h x) \psi(x)$ vanishes outside a compact set in $(-\Lambda, \Lambda)$. But this is obvious since the transform is $h^{-1} \hat{\alpha}\left(\xi h^{-1}\right) * \hat{\psi}(\xi)$.

Now we have $\alpha(h x) f^{(n)}(x) \leqq M_{2}$ and $\alpha(h x) f^{(n)}(x)$ is also bounded from below by some number $-M_{1}$. Hence, if $h<\Lambda$ there exists a function $f_{h}(x)$ with no spectrum in $(-(\Lambda-h),(\Lambda-h))$ such that $f_{h}^{(n)}(x)=\alpha(h x) f^{(n)}(x)$ and

$$
\frac{-\mu_{2}^{(n)}\left(\infty, M_{2}\right)}{(\Lambda-h)^{n}} \leqq-\frac{\mu_{1}^{(n)}\left(M_{1}, M_{2}\right)}{(\Lambda-h)^{n}} \leqq f_{h}(x) \leqq \frac{\mu_{2}^{(n)}\left(M_{1}, M_{2}\right)}{(\Lambda-h)^{n}} \leqq \frac{\mu_{2}^{(n)}\left(\infty, M_{2}\right)}{(\Lambda-h)^{n}}
$$

We conclude with the same argument as in the proof of theorem 2 above that $f_{h}(x) \rightarrow f(x)$ when $h \rightarrow 0$. Hence we get the inequality

$$
-\Lambda^{-n} \mu_{1}^{(n)}\left(\infty, M_{2}\right) \leqq f(x) \leqq \Lambda^{-n} \mu_{2}^{(n)}\left(\infty, M_{2}\right) .
$$

In the general case choose any $\beta(x) \in \mathscr{P}$ such that $\beta(x) \geqq 0$ and $\int_{-\infty}^{+\infty} \beta(x) d x=1$. Form 


$$
f_{\beta}(x)=\beta * f(x)=\int_{-\infty}^{+\infty} \beta(x-t) f(t) d t=\int_{-\infty}^{+\infty} \beta(t) f(x-t) d t
$$

We have

$$
f_{\beta}^{(n)}(x)=\int_{-\infty}^{+\infty} \beta^{(n)}(x-t) f(t) d t
$$

and hence

$$
\left|f^{(n)}(x)\right| \leqq \int_{-\infty}^{+\infty} C\left(1+(x-t)^{2}\right)^{N}\left|\beta^{(n)}(t)\right| d t
$$

where the right hand side of the inequality is a polynomial in $x$. On the other hand we can write $f_{\beta}^{(n-1)}(x)=\int_{-\infty}^{+\infty} f^{(n-1)}(x-t) \beta(t) d t$ and hence

$$
\frac{f_{\beta}^{(n-1)}(x)-f_{\beta}^{(n-1)}(y)}{x-y} \leqq \int_{-\infty}^{+\infty} M_{2} \beta(t) d t=M_{2} .
$$

No part of the spectrum of $f_{\beta}(x)$ belongs to $(-\Lambda, \Lambda)$. For if $\psi(x) \in \mathcal{f}$ and $\hat{\psi}(\xi)=0$ outside a compact set in $(-\Lambda, \Lambda)$, we have

$$
\int_{-\infty}^{+\infty} f_{\beta}(x) \psi(x) d x=\int_{-\infty}^{+\infty} f(t) d t \int_{-\infty}^{+\infty} \beta(x-t) \psi(x) d x=0
$$

since the Fourier transform of $\int_{-\infty}^{+\infty} \beta(x-t) \psi(t) d t$ is $\hat{\beta}(-\xi) \hat{\psi}(\xi)$ and this vanishes outside a compact set in $(-\Lambda, \Lambda)$.

We can now conclude, from the case treated first, that

$$
-\Lambda^{-n} \mu_{1}^{(n)}\left(\infty, M_{2}\right) \leqq f_{\beta}(x) \leqq \Lambda^{-n} \mu_{2}^{(n)}\left(\infty, M_{2}\right)
$$

Hence, letting $\beta$ converge to the Dirac measure at the origin, we get

$$
-\Lambda^{-n} \mu_{1}^{(n)}\left(\infty, M_{2}\right) \leqq f(x) \leqq \Lambda^{-n} \mu_{2}^{(n)}\left(\infty, M_{2}\right)
$$

and the theorem is proved.

The explicit value of $\mu_{i}^{(n)}$. By lemma 2 we know that $h_{n}{ }^{\prime}(x)$ has only those two zeros which correspond to the maximum and the minimum of $h_{n}(x)$. Hence to calculate $\mu_{i}^{(n)}$ we have only to find the two zeros of $h_{n}{ }^{\prime}(x)=h_{n-1}(x)$. This is easy if $n$ is even, $n=2 k$. For from (5) it follows immediately that $h_{2 k}{ }^{\prime}(0)=h_{2 k}{ }^{\prime}\left(\frac{1}{2}\right)=0$ on account of the symmetry properties of $\bar{B}_{2 k}(x)$. To determine whether $x=0$ is a maximum or a minimum we first note that $h_{2}(x)$ has a maximum at the origin. Hence $h_{4}{ }^{\prime \prime}(0)=h_{2}(0)>0$ so that $h_{4}(x)$ has a minimum at the origin. In this way we immediately prove that $h_{2 k}(x)$ has a maximum for $x=0$ if $k$ is 
odd and a minimum if $k$ is even. It is clear that $x=\frac{1}{2}$ has the opposite extremal character. Now we have

$$
\begin{gathered}
h_{2 k}(0)=2 \frac{M_{1}+M_{2}}{(2 k+1) !} \bar{B}_{2 k+1}\left(\frac{M_{2}}{2\left(M_{1}+M_{2}\right)}\right), \\
h_{2 k}\left(\frac{1}{2}\right)=2 \frac{M_{1}+M_{2}}{(2 k+1) !} \bar{B}_{2 k+1}\left(\frac{M_{2}}{2\left(M_{1}+M_{2}\right)}+\frac{1}{2}\right),
\end{gathered}
$$

and we may drop the bar over $B$ here because the arguments are in the interval $(0,1)$. These formulae can be written differently if we use two well-known formulae from the calculus of finite differences (cf. Nörlund [6, Kapitel 2-3]): $B_{n}(x)+B_{n}\left(x+\frac{1}{2}\right)=2^{1-n} B_{n}(2 x), B_{n}\left(x+\frac{1}{2}\right)-B_{n}(x)=$ $2^{-n} n E_{n-1}(2 x)$ where $E_{n-1}$ is an Eulerian polynomial. We get

$$
h_{2 k}(0)=\frac{M_{1}+M_{2}}{2^{2 k+1}}\left\{\frac{2}{(2 k+1) !} B_{2 k+1}\left(\frac{M_{2}}{M_{1}+M_{2}}\right)-\frac{1}{(2 k) !} E_{2 k}\left(\frac{M_{2}}{M_{1}+M_{2}}\right)\right\}
$$

and the same expression for $h_{2 k}\left(\frac{1}{2}\right)$ except that there is a plus instead of the minus sign. Combining this with the discussion of the extremal character of $x=0$ and $x=\frac{1}{2}$ performed above, we get the perfectly symmetric formula

$$
\begin{gathered}
\mu_{i}^{(2 k)}=\frac{M_{1}+M_{2}}{2^{2 k+1}}\left\{\frac{2}{(2 k+1) !} B_{2 k+1}\left(\frac{M_{i}}{M_{1}+M_{2}}\right)+\frac{(-1)^{k}}{(2 k) !} E_{2 k}\left(\frac{M_{i}}{M_{1}+M_{2}}\right)\right\}, \\
i=1,2, \quad k=1,2, \ldots
\end{gathered}
$$

For $n$ odd the maxima and minima cannot be found explicitly except in the case of Favard, where $M_{1}=M_{2}$, and in the case where $n$ is so small that we can solve the equation $h_{n-1}(x)=0$. Thus, for $n=1$ we easily find from a figure:

$$
\mu_{1}^{(1)}=\mu_{2}^{(1)}=\frac{M_{1} M_{2}}{2\left(M_{1}+M_{2}\right)}
$$

that is, one fourth of the harmonic mean of $M_{1}$ and $M_{2}$.

An inequality of Kolmogoroff. There exists a strong connection between the inequality of Bohr and an inequality of Kolmogoroff (Kolmogoroff [4], Bang [1]), and we therefore outline a generalization of the latter, too. Put

Let $f(x)$ be a real function on $(-\infty,+\infty)$ with $n$ continuous derivatives. 


$$
M_{1}{ }^{(k)}=-\inf _{-\infty<x<\infty} f^{(n-k)}(x), \quad M_{2}{ }^{(k)}=\sup _{-\infty<x<\infty} f^{(n-k)}(x) .
$$

Suppose that $M_{1}{ }^{(n)}, M_{2}{ }^{(n)}$ and at least one of $M_{1}{ }^{(0)}$ and $M_{2}{ }^{(0)}$ are finite. It follows at once that $M_{1}{ }^{(0)}$ and $M_{2}{ }^{(0)}$ are non negative. The following generalization of Kolmogoroff's inequality is valid:

$$
\left(\frac{M_{i}{ }^{(k)}}{\mu_{i}{ }^{(k)}\left(M_{1}{ }^{(0)}, M_{2}{ }^{(0)}\right)}\right)^{1 / k} \leqq\left(\frac{M_{1}^{(n)}+M_{2}^{(n)}}{\mu_{1}^{(n)}\left(M_{1}{ }^{(0)}, M_{2}{ }^{(0)}\right)+\mu_{2}^{(n)}\left(M_{1}{ }^{(0)}, M_{2}{ }^{(0)}\right)}\right)^{1 / n} .
$$

There is equality for the function

$$
\alpha^{-n} h_{n}\left(\alpha x ; M_{1}{ }^{(0)}, M_{2}{ }^{(0)}\right)+\beta \quad(\alpha>0),
$$

where $\alpha$ and $\beta$ are chosen so that the maximum and minimum of this function are $M_{2}{ }^{(n)}$ and $-M_{1}{ }^{(n)}$ respectively. However, we shall not give a detailed proof, because the ideas of [1] and [4] are sufficient in this more general case.

\section{BIBLIOGRAPHY}

1. Th. Bang, Une inégalité de Kolmogoroff et les fonctions presque-périodiques, Danske Vid. Selsk. Mat.-Fys. Medd. XIX, 4, 1941.

2. H. Bohr, Ein allgemeiner Satz über die Integration eines trigonometrischen Polynoms, Prace Matem.-Fiz. 43 (1935), 273-288 (= Collected Mathematical Works II, C 36).

3. J. Favard, Application de la formule sommatoire d'Euler à la démonstration de quelques propriétés extrémales des intégrales des fonctions périodiques et presque-périodiques, Mat. Tidsskr. B 1936, 81-94.

4. A. N. Kolmogoroff, On inequalities between upper bounds of consecutive derivatives of an arbitrary function defined on an infinite interval, Učenye Zapiski Moskov. Gos. Univ. Matematika 30 (1939), 3-16; Amer. Math. Soc. Translation 4.

5. B. M. Lewitan, Über eine Verallgemeinerung der Ungleichungen von S. Bernstein und H. Bohr, Doklady Akad. Nauk SSSR 15 (1937), 169-172.

6. N. E. Nörlund, Vorlesungen über Differenzenrechung, Berlin, 1924.

7. L. Schwartz, Théorie des distributions II, Paris, 1951. 